\title{
LIGHT ENGINE AND OPTICS FOR HELIUM3D AUTO-STEREOSCOPIC LASER SCANNING DISPLAY
}

\author{
Kaan Akşit, Selim Ölçer, Erdem Erden, Kishore V.C., Hakan Urey \\ Koç University, Department of Electronic \& Electrical Engineering, Istanbul, Turkey* \\ Eero Willman, Hadi Baghsiahi, Sally E. Day, David R. Selviah, F. Aníbal Fernández \\ University College London, Department of Electronic \& Electrical Engineering, London, U.K.
}

\section{Phil Surman}

De Montfort University, Department of Computer Technology, Leicester, U.K.

\begin{abstract}
This paper presents a laser based auto-stereoscopic 3D display technique and a prototype utilizing a dual projector light engine. The solution described is able to form dynamic exit pupils under the control of a multi-user head-tracker. A prototype completed recently is able to provide a glasses-free solution for a single user at a fixed position. At the end of the prototyping phase it is expected to enable a multiple user interface with an integration of the pupil tracker and the spatial light modulator.
\end{abstract}

Index Terms - 3D Display, laser scanning, pupil tracking, auto-stereoscopic, dynamic exit pupil

\section{INTRODUCTION}

European union seventh framework programme funded project, HELIUM3D (High Efficiency Laser-Based Multiuser Multi-modal 3D Display) is made up a consortium that includes Universities and institutes; Koç University from Turkey, De Montfort University and University College London from the UK, Nanjing University from China, Barco from Belgium, Fraunhofer HHI from Germany, Philips and Eindhoven University of Technology from the Netherlands. The main goal of the project is to enable the technology to exceed the limits of existing stereoscopic display techniques. The main contribution will be as follows: enabling of a glasses-free stereoscopic display technique, more efficient usage of light sources via sophisticated optical setup and enhancement of picture quality with the use of laser light sources. Until now, there have been several publications reviewing the concept and updating its status, [1], [2] [3], [4],[5] and [6]. The interest of the market in 3D display technologies has increased in the past

*Funding from the European Union FP7 for the HELIUM3D project (contract No. 215280) is acknowledged. All the authors of the paper would like to thank all the members of the HELIUM3D group. years. Currently most of the display manufacturers provide 3D displays with shutter glasses.

\section{DESCRIPTION OF THE SYSTEM}

As illustrated in Figure 1, whole system consists of three principal parts, these are: the light engine, the transfer screen and the head tracker. These are described in detail in the various papers cited.

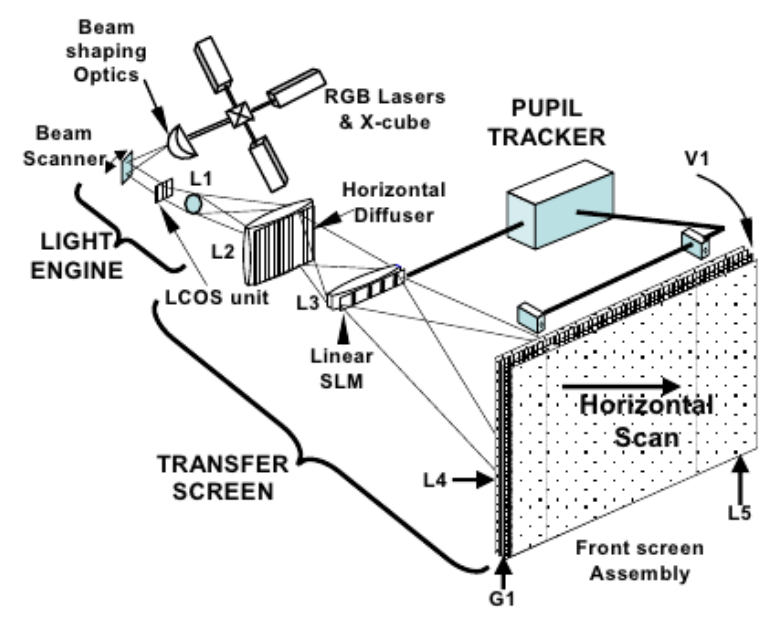

Fig. 1. A simplified schematic diagram showing the subsystems comprising the HELIUM3D display, [5].

The light engine consists of the light source, the beam shaping optics, a scanner mirror and two Liquid-Crystal-onSilicon (LCoS) projectors. Red, green and blue (RGB) lasers are used as light sources. The three laser sources are combined via an X-Cube prism that is taken out of a conventional projector. Combined light is sent into the beam shaping optics. The aim is to create a uniform white line at the LCoS 
planes. This line is scanned on to LCoS unit using a scanning mirror that is fed by a $60 \mathrm{~Hz}$ saw-tooth drive signal. The pupil tracker unit is capable of tracking and locating a user's head and eye position in real-time as $(\mathrm{x}, \mathrm{y})$ coordinates. Information coming from the pupil tracker is fed into the Spatial Light Modulator (SLM). The transfer screen is discussed in the next section.

\section{TRANSFER SCREEN}

The prototype shown in Figure 6 is a simplified version of the final prototype where the principal difference is the use of shutter glasses to provide the function of the SLM. The shutter glasses shown in Figure 2 produce fixed exit pupils; these are regions in the viewing field where a left or a right image is seen by the viewer. The purpose of the transfer screen is to enable the directions of the rays exiting the viewing screen to be controlled so that exit pupils are directed to preferred locations.

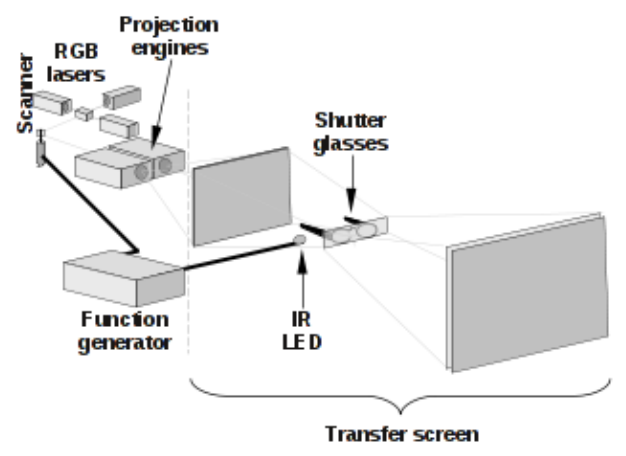

Fig. 2. Schematic diagram showing the shutter glasses used to control the light directions from the front screen.

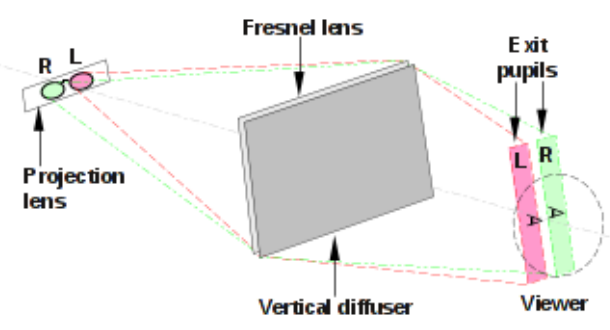

Fig. 3. Exit pupil formation with Fresnel lens and vertical diffuser.

Figure 3 shows the way in which exit pupils are formed with the use of a spherical Fresnel lens and a vertical diffuser. If the screen consisted of a lens only, small exit pupils that are real images of the shutter glass filters would be formed. In order to give viewers vertical movement these images are expanded into the regions shown in the right side of the figure with the use of a lenticular screen acting as a vertical only diffuser. Stereo is presented by displaying an image in the right projection engine and allowing the shutter glass filter corresponding to the right eye to transmit light with the left filter off. During the following frame an image is displayed on the left projector and the left shutter glass filter transmits with the right filter off. In this way left and right images are presented sequentially at the left and right exit pupils. As the scanner directs illumination to the left and right projectors alternately in a single scan the shutter glasses must run in synchronization; this is achieved by controlling the shutter glasses with an infra red LED that runs from the same function generator as the scanner. The object of the HELIUM3D display is to provide more than one pair of exit pupils so that several viewers can be accommodated and exit pupil movement in both the $X$ and $\mathrm{Z}$ directions and viewers are able to move around over a large area. The performance of this prototype is limited and to address this, the final version will incorporate the following:

- Replace the Fresnel lens with a Gabor superlens to give a wider viewing field.

- Use a linear SLM with comprising 256 elements instead of the shutter glasses. This enables several exit pupil pairs to be produced that can be steered in the $X$ and $\mathrm{Z}$ directions under the control of a multi-user head position tracker.

\section{CURRENT PROTOTYPE}

Currently the existing prototype has some deviations from the designed system that was introduced in Section 2. As discussed in Section 2, RGB laser light sources are used. Two different sets of laser sources are used as light sources in experiments. The first set consists of controllable low power single-emitter laser light sources $(100 \sim 200 \mathrm{~mW})$. The second set consists of controllable high power multi-emitter $(2 \times$ 24) laser light sources from Arasor (3000 $4000 \mathrm{~mW})$. but the current prototype uses the first set but in the near future experiments of the whole system with second set will be made; this is expected to have a brighter and more uniform image at the user plane.

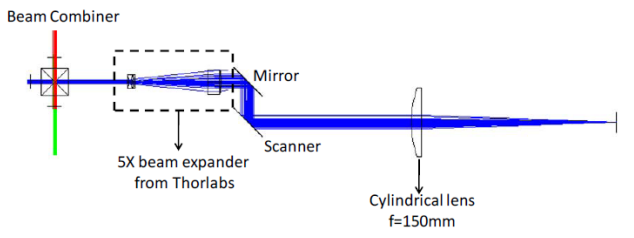

Fig. 4. ZEMAX design of the light engine of the prototype.

Unlike Figure 4, the existing prototype combines three light sources using dichroic mirrors instead of an X-Cube. The prototype consists of two LCoS units, but rest of the 
used optics are the same. Taken from conventional projectors the $60 \mathrm{~Hz}$ projection engines are used to provide $120 \mathrm{~Hz}$ frame rate by displaying their outputs alternately in $16.7 \mathrm{~ms}$ period. The scanned beam inputs both projectors in single sweep. Figure 5 shows how three colors are combined and fed into dual LCoS units. It should be noted that there is a group of mirrors at the entrance of the LCoS units; these are used to separate the two input paths during the sweep.

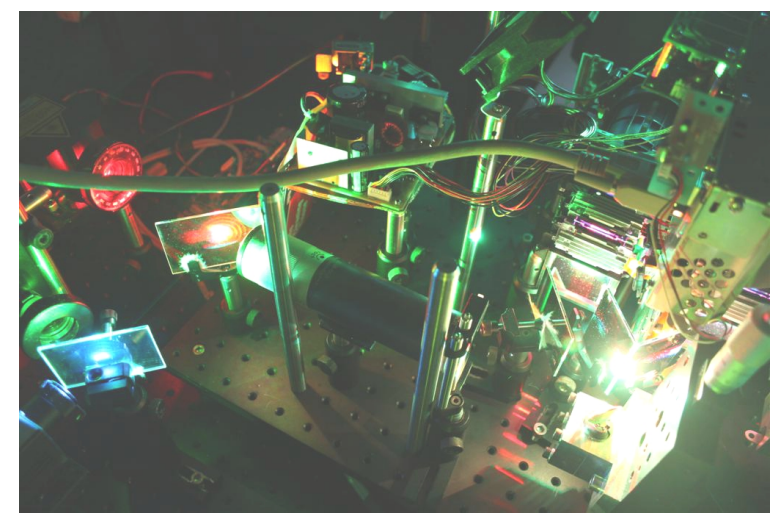

Fig. 5. Low power laser set combined using dichroic mirrors and projected into two LCoS units using mirrors.

After existing the LCoS units the light enters the transfer screen section. One major difference between the existing prototype ant the final system is the SLM; currently, a pair of NVIDIA 3D Vision's shutter glasses is in used as the SLM. The drive signals of mirror-scanner and the shutter glasses are provided from the same function generator, so that, both signals are synchronized. Beside this hardware implementation of the synchronization, software has been developed to control the shutter glasses without generating the drive signal externally, see [7]. Synchronization is necessary to decrease the ghosting effect to a negligible level. and during experiments this has not been noticeable. Figure 6 shows a photograph of the existing prototype operating for a single user.

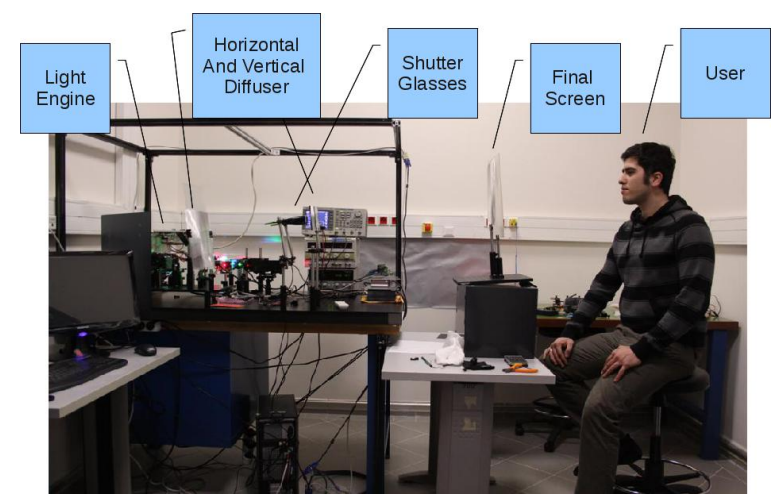

Fig. 6. A photograph of the whole system operating for a single user.
In addition to static images the current system can also demonstrate stereo video content. It was observed that playing a movie image gives more immunity against imperfections in the setup such as imperfect color balance or other image artifacts.

\section{RECENT RESULTS}

In addition to the existing prototype, experiments with high power lasers are continuing. The goal is to replace the first set of lasers with the high power versions. Another setup has been developed to examine the performance of the system before the lasers are replaced. Figure 7 shows the setup with second high power set where RGB laser sources are combined via a dichroic mirrors. The combined white light is coupled to a fiber whose output illuminates the beam shaping optics consisting of micro-lens arrays.

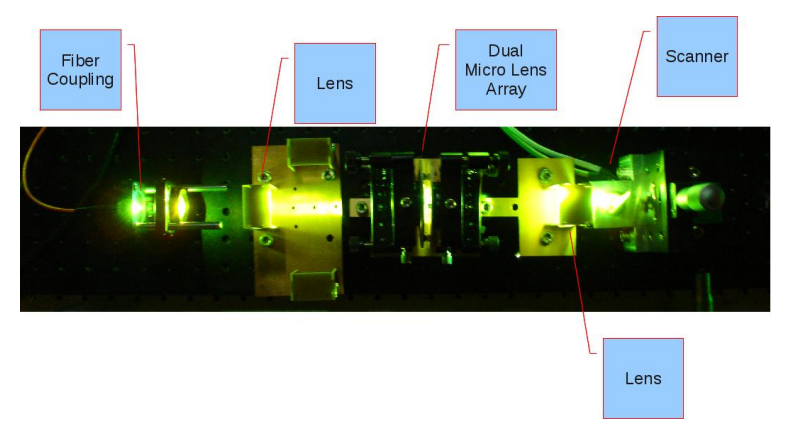

Fig. 7. A photograph of the beam shaping optics combined with fiber coupling and the scanner.

As shown in Figure 8a, the distribution of the light after the fiber coupling at LCoS plane has a homogeneous light density distribution. It was observed that the system without the fiber coupling resulted with a strong variation in light density at LCoS plane and can be observed with the naked eyes. The variation without the fiber coupling is caused by a varying pattern of periodic brighter and darker lines at the LCoS plane due to diffraction artifacts. Therefore, the result with the fiber coupling can be concluded as an important milestone for the project as this gives improved image quality and the brightness at the user plane. Figure 9 shows the light density distribution of the Figure $8 b$. 


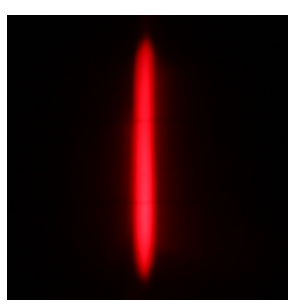

(a) A single line at $\mathrm{LCoS}$ plane.

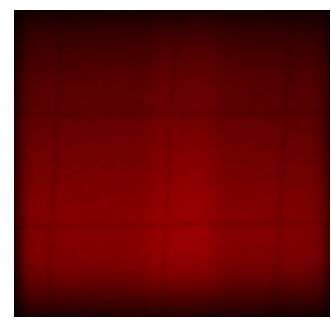

(b) Scanned light on a piece of paper at the LCoS plane with saw-tooth scanner drive input.
Fig. 8. Light density distribution at the LCoS plane with and without scanning.

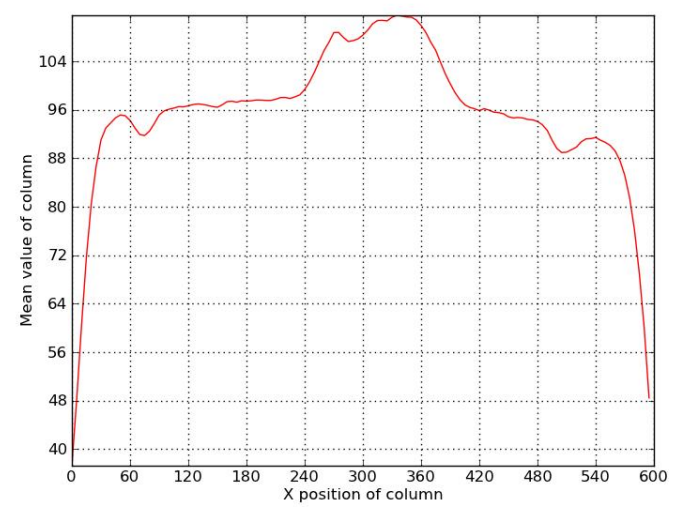

Fig. 9. The light density distribution in $\mathrm{X}$-axis of Figure $8 \mathrm{~b}$.

The intensity variations in Figure 9 can be improved further by adjusting the micro-lens arrays configuration.

\section{CONCLUSION}

The most recent results from the experiment bench has been presented. It is shown that the prototype is capable of 3D display for a single user. Effective light source usage enables the prototype to operate in well-illuminated environments such as homes and offices so that the one of the project's goals is reached. At this time it has been demonstrated that the current prototype is close to providing a good auto-stereoscopic 3D display system. There are still some parts to be integrated in order to finalize the prototype and evaluate this. The final prototype will incorporate a high power RGB, laser set with fiber coupled beam shaping optics, an SLM and pupil tracker unit and improved transfer screen. The most challenging component is the SLM which must be fast with a response time of around $160 \mu \mathrm{s}$ and comprise 256 elements in a linear array. This is currently under development and is expected to be incorporated into the prototype in the near future.

\section{REFERENCES}

[1] I. Sexton, R. Bates, W. Lee, P. Surman, K. Hopf, F. Neumann, A. Corbett, and E. Buckley, "Laser illuminated multi-viewer 3D displays," Proc. IMID 2008, pp14231426, Seoul, 2008.

[2] R.S. Brar, P. Surman, and I. Sexton, "Projection-based head-tracking 3-D displays," Journal of the Society for Information Display, vol. 18, pp. 844, 2010.

[3] P. Surman, I. Sexton, K. Hopf, W.K. Lee, E. Buckley, G. Jones, and R. Bates, "European Research into Head Tracked Autostereoscopic Displays," in 3DTV Conference: The True Vision-Capture, Transmission and Display of 3D Video, 2008. IEEE, 2008, pp. 161-164.

[4] H. Urey, VC Kishore, E. Erden, and P. Surman, "State of the Art in Stereoscopic and Autostereoscopic Displays," in Proceedings of the IEEE, April 2011.

[5] E. Erden, VC Kishore, H. Urey, H. Baghsiahi, E. Willman, S.E. Day, D.R. Selviah, F.A. Fernandez, and P. Surman, "Laser scanning based autostereoscopic 3D display with pupil tracking," in LEOS Annual Meeting Conference Proceedings, 2009. LEOS'09. IEEE. IEEE, 2009, pp. 10-11.

[6] E. Willman, H. Baghsiahi, FA Fernández, DR Selviah, SE Day, VC Kishore, E. Erden, H. Urey, and PA Surman, "The optics of an autostereoscopic multiview display," The Society for information Display, International Symposium, Seminar and Exhibition, Washington State Convention Center, Seattle, WA, USA, 2010.

[7] Kaan Akşit, "pyusbir: A python based module to operate the usb ir device of nvidia shutter glasses," http: // code.google.com/p/pyusbir/, Jan. 2011. 